PASJ: Publ. Astron. Soc. Japan , 1-??,

(C) 2018. Astronomical Society of Japan.

\title{
The First MAXI/GSC Catalog in the High Galactic-Latitude Sky
}

\author{
Kazuo Hiror ${ }^{1}$ Yoshihiro Ueda, ${ }^{1}$ Naoki Isobe,${ }^{1,2}$ Masaaki Hayashida, ${ }^{1}$ Satoshi Eguchi,${ }^{1,3}$ Mutsumi Sugizaki, ${ }^{4}$ \\ Nobuyuki Kawai,${ }^{5}$ Hiroshi Tsunemi,${ }^{6}$ Masaru Matsuoka,,${ }^{4,7}$ Tatehiro Minara, ${ }^{4}$ Kazutaka Yamaoka, ${ }^{8}$ Masaki \\ Ishikawa, ${ }^{9}$ Masashi Kimura, ${ }^{6}$ Hiroki Kitayama,${ }^{6}$ Mitsuhiro Kohama,${ }^{7}$ Takanori Matsumura, ${ }^{10}$ Mikio MoriI ${ }^{5}$ \\ Yujin E. Nakagawa, ${ }^{11}$ Satoshi Nakahira, ${ }^{4}$ Motoki Nakajima, ${ }^{12}$ Hitoshi Negoro, ${ }^{13}$ Motoko Serino, ${ }^{4}$ Megumi \\ Shidatsu, ${ }^{1}$ Tetsuya Sootome, ${ }^{4}$ Kousuke Sugimori,${ }^{5}$ Fumitoshi Suwa, ${ }^{13}$ Takahiro Toizumi, ${ }^{5}$ Hiroshi Tomida, ${ }^{7}$ \\ Yohko Tsubor ${ }^{10}$ Shiro Ueno,${ }^{7}$ Ryuichi Usui,${ }^{5}$ Takayuki Yamamoto, ${ }^{4}$ Kyohei Yamazaki, ${ }^{10}$ Atsumasa YoshidA, ${ }^{8}$ \\ and the MAXI team \\ ${ }^{1}$ Department of Astronomy, Kyoto University, Oiwake-cho, Sakyo-ku, Kyoto 606-8502 \\ hiroi@kusastro.kyoto-u.ac.jp \\ ${ }^{2}$ Institute of Space and Astronautical Science (ISAS), Japan Aerospace Exploration Agency (JAXA) ,3-1-1 Yoshino-dai, \\ Chuo-ku, Sagamihara, Kanagawa 252-5210 \\ ${ }^{3}$ National Astronomical Observatory of Japan, 2-21-1, Osawa, Mitaka City, Tokyo 181-8588 \\ ${ }^{4}$ MAXI team, Institute of Physical and Chemical Research (RIKEN), 2-1 Hirosawa, Wako, Saitama 351-0198 \\ ${ }^{5}$ Department of Physics, Tokyo Institute of Technology, 2-12-1 Ookayama, Meguro-ku, Tokyo 152-8551 \\ ${ }^{6}$ Department of Earth and Space Science, Osaka University, 1-1 Machikaneyama, Toyonaka, Osaka 560-0043 \\ ${ }^{7}$ ISS Science Project Office, Institute of Space and Astronautical Science (ISAS), Japan Aerospace Exploration Agency \\ (JAXA), 2-1-1 Sengen, Tsukuba, Ibaraki 305-8505 \\ ${ }^{8}$ Department of Physics and Mathematics, Aoyama Gakuin University, \\ 5-10-1 Fuchinobe, Chuo-ku, Sagamihara, Kanagawa 252-5258 \\ ${ }^{9}$ School of Physical Science, Space and Astronautical Science, The graduate University for Advanced Studies (Sokendai), \\ Yoshinodai 3-1-1, Chuo-ku, Sagamihara, Kanagawa 252-5210 \\ ${ }^{10}$ Department of Physics, Chuo University, 1-13-27 Kasuga, Bunkyo-ku, Tokyo 112-8551 \\ ${ }^{11}$ Research Institute for Science and Engineering, Waseda University, 17 Kikui-cho, Shinjuku-ku, Tokyo 162-0044 \\ ${ }^{12}$ School of Dentistry at Matsudo, Nihon University, 2-870-1 Sakaecho-nishi, Matsudo, Chiba 101-8308 \\ ${ }^{13}$ Department of Physics, Nihon University, 1-8-14 Kanda-Surugadai, Chiyoda-ku, Tokyo 101-8308
}

(Received 2011 June 12; accepted 2011 August 13)

\begin{abstract}
We present the first unbiased source catalog of the Monitor of All-sky X-ray Image (MAXI) mission at high Galactic latitudes $\left(|b|>10^{\circ}\right)$, produced from the first 7-month data (2009 September 1 to 2010 March 31) of the Gas Slit Camera in the 4-10 keV band. We develop an analysis procedure to detect faint sources from the MAXI data, utilizing a maximum likelihood image fitting method, where the image response, background, and detailed observational conditions are taken into account. The catalog consists of 143 X-ray sources above 7 sigma significance level with a limiting sensitivity of $\sim 1.5 \times 10^{-11} \mathrm{ergs} \mathrm{cm}^{-2} \mathrm{~s}^{-1}$ $(1.2 \mathrm{mCrab})$ in the $4-10 \mathrm{keV}$ band. Among them, we identify 38 Galactic/LMC/SMC objects, 48 galaxy clusters, 39 Seyfert galaxies, 12 blazars, and 1 galaxy. Other 4 sources are confused with multiple objects, and one remains unidentified. The $\log N-\log S$ relation of extragalactic objects is in a good agreement with the HEAO-1 A-2 result, although the list of the brightest AGNs in the entire sky has significantly changed since that in 30 years ago.
\end{abstract}

Key words: catalogs - surveys — galaxies: active - X-rays: galaxies

\section{INTRODUCTION}

All-sky X-ray surveys are powerful tools to investigate the whole populations of active and hot phenomena in the universe at the brightest flux end. The strong Xray emitters include Galactic objects such as active stars, SNRs, pulsars, CVs, low mass and high mass X-ray binaries (with a neutron star or a black hole as the primary), and extragalactic objects, mainly active galactic nuclei (AGNs; Seyfert galaxies and blazars) and clusters of galaxies. The source catalog consisting of a statistically well-defined sample detected from an unbiased survey is a primary product on which many subsequent studies are based. For extragalactic populations, in particular, these results define the "local" sample in the present universe, the end point of their cosmological evolution. Thus, to establish the statistical properties of bright X-ray sources using the best quality data over the entire sky has always been a key issue in high energy astrophysics.

Past all-sky X-ray surveys indeed brought valuable information on the X-ray source populations. In the soft $\mathrm{X}$-ray band, the ROSAT mission conducted an all-sky survey in the $0.1-2.4 \mathrm{keV}$ band, producing the ROSAT AllSky Survey (RASS) Bright Source Catalog (BSC; Voges 
et al. 1999) and Faint Source Catalog (FSC; Voges et al. 2000), which contain 18,811 and 105,924 sources, respectively. Because of its large sample size, only a part of the RASS sources has been optically identified (e.g., Schwope et al. 2000). Hard X-rays above $2 \mathrm{keV}$ are more effective to detect obscured objects, such as "type 2" AGNs, due to its strong penetrating power against photoelectric absorption. In the late 1970s, HEAO-1 A-2 performed an all-sky X-ray survey at $|b|>20^{\circ}$ in the $2-10 \mathrm{keV}$ band down to a limiting sensitivity of $\sim 3.1 \times 10^{-11} \mathrm{ergs} \mathrm{cm}^{-2}$ $\mathrm{s}^{-1}$, detecting 61 extragalactic sources including 29 AGNs (Piccinotti et al. 1982). Rossi X-ray Timing Explorer (RXTE) also carried out an all-sky survey using "slew" mode data of the Proportional Counter Array (PCA) in the $3-8 \mathrm{keV}$ and $8-20 \mathrm{keV}$ bands, and achieved the sensitivity similar to, and an order of magnitude higher than those of HEAO-1 A-1 and A-4, respectively (Revnivtsev et al. 2004). The RXTE/PCA survey detected 294 sources including $100 \mathrm{AGNs}$, although the identification is not complete (80\%). Recently, the Swift and INTEGRAL satellites have performed all-sky surveys in the hard X-ray band above $10 \mathrm{keV}$ (Swift: Tueller et al. 2008; Tueller et al. 2010; Cusumano et al. 2010; Baumgartner et al. 2010, INTEGRAL: Bird et al. 2007; Beckmann et al. 2006, 2009; Krivonos et al. 2007; Bird et al. 2010). These catalogs contain heavily obscured AGNs with absorption column densities larger than $10^{24} \mathrm{~cm}^{-2}$. Total 628 AGNs have been detected in the Palermo Swift/BAT 54-month catalog (Cusumano et al. 2010).

Monitor of All-sky X-ray Image (MAXI; Matsuoka et al. 2009 ) is the first scientific mission operated on the international space station (ISS). It carries two types of X-ray cameras: Gas Slit Camera (GSC; Sugizaki et al. 2011; Mihara et al. 2011) and Solid-state Slit Camera (SSC; Tsunemi et al. 2010; Tomida et al. 2011), covering the energy bands of 2-30 keV and $0.5-12 \mathrm{keV}$, respectively. MAXI/GSC observes nearly the whole sky every 92 minutes with two instantaneous fields of view of $160^{\circ} \times 3^{\circ}$. One of the main goals of the MAXI mission is to provide a new all-sky X-ray source catalog, including both transient and persistent objects. By integrating the data over a long period, MAXI/GSC is expected to achieve so far the best sensitivities as an all-sky mission that covers the 2-10 keV band (Matsuoka et al. 2009, Ueda et al. 2010). Since its energy band is complementary to that of the RASS (below $2 \mathrm{keV}$ ) and to those of Swift/BAT and INTEGRAL (above $10 \mathrm{keV}$ ), it will have an advantage in detecting sources having an intrinsically soft continuum with moderate absorption.

In this paper, we present the first MAXI/GSC source catalog detected in the $4-10 \mathrm{keV}$ band at high Galactic latitudes $\left(|b|>10^{\circ}\right)$, utilizing the first 7-month data since the start of its nominal operation. The data reduction and filtering are given in section 2. Section 3 describes the background model of MAXI/GSC used in the analysis and the details of image analysis procedure. In section 4, we present the source catalog and summarize the X-ray properties, results of cross correlation with other catalogs and identification, position accuracy, and $\log N-\log S$ relations. Section 5 gives the conclusion. The analysis of the local luminosity function of Seyfert galaxies based on this catalog is reported in an accompanying paper (Ueda et al. 2011, submitted to PASJ).

\section{DATA REDUCTION}

For the catalog production, we use the MAXI/GSC data taken between 2009 September 1 and 2010 March 31 , when all the GSC counters were operated with a high voltage of $1650 \mathrm{~V}$. Only the data in the $4-10 \mathrm{keV}$ band are utilized in this paper, because in this energy band (1) the energy and position responses are best calibrated at present, and (2) a high signal-to-noise ratio is achieved thanks to high detection efficiency of the counters and to relatively low background rate (Sugizaki et al. 2011).

Starting from the event files with processing version 0.3 provided by the MAXI team, we apply the following data screening to obtain clean data used in the image analysis described in section 3. The processed event files contain columns of the arrival time (TIME), energy (PI), and sky position (R.A. and Dec. ) for each photon as essential information. We utilize the data of all the twelve GSC counters from 2009 September 1 to 22, while those of only eight counters (GSC_0, 1, 2, 3, 4, 5, 7, and 8) are included in the later epoch since the operation of the rest four counters were stopped due to a hardware trouble. The photon events detected by the carbon anodes \#1 and \#2 in all the counters are excluded because of the response problem in the current calibration. To discard the data suffering from high background rates, we only utilize the data taken when the ISS latitude is between $-40^{\circ}$ and $40^{\circ}$, and those detected at the central part of each counter with the photon incident angle $|\phi|<38^{\circ}$ ( $\phi$; for definition, see Mihara et al. 2011).

Figure 1 displays the effective exposure map in the Galactic coordinates for the MAXI/GSC 7-month data, where the net exposure corrected for the detection efficiency, multiplied by the projected area of the slit $(\alpha$ $\cos \phi$ ), is given in units of $\mathrm{s} \mathrm{cm}{ }^{2}$ at each sky position. This is obtained from the simulation utilizing the MAXI simulator maxisim (Eguchi et al. 2009) by assuming a uniformly extended emission as the input source; hence this plot is inevitably smoothed by $\sim 3$ degree, an angular resolution of MAXI.

\section{ANALYSIS}

To detect X-ray sources in an unbiased way from the MAXI/GSC data, we perform an image analysis where the image response, background, and detailed observational conditions are taken into account. We first examine the properties of the MAXI/GSC background on the basis of the on-board data, and attempt to construct a background model (section 3.1). Then, in section 3.2, we employ a two-step approach to search for source candidates, and determine their fluxes and positions. 


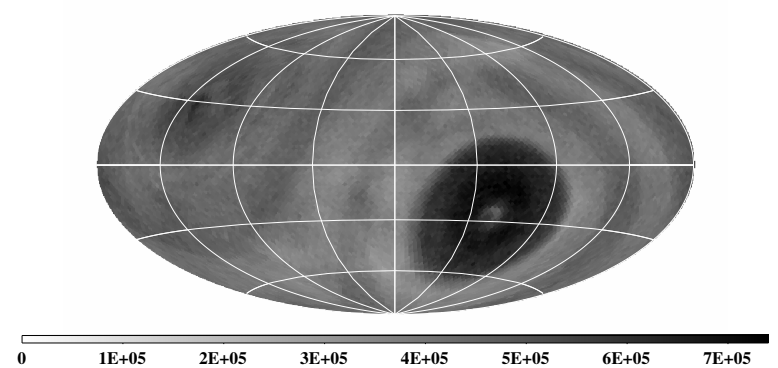

Fig. 1. The effective exposure map for the 7-month MAXI/GSC data in the Galactic coordinates projected with the Aitoff algorithm. The unit is $\mathrm{s} \mathrm{cm}^{2}$. The black annular-like structures at the bottom-right and top-left positions correspond to regions of the longest exposure near the two poles of the rotation axis of the ISS's orbital motion.

\subsection{Background Reproduction}

In order to securely detect faint X-ray sources with a minimum number of fake detections and to determine their X-ray fluxes precisely, it is of crucial importance to reproduce the instrumental non-X-ray background (NXB) level with a high accuracy. Thus, we model the MAXI/GSC background as a function of time, on the basis of the observational properties. In the case of MAXI, it is difficult to disentangle the cosmic X-ray background (CXB) and NXB from the on-board data, since MAXI rarely observes a direction of the night earth that blocks the CXB. Therefore, we here consider the sum of these two background components.

First, we examine the long-term variability of the background. For this purpose, we make daily averaged background count rate for each counter by rejecting periods when bright X-ray point sources or the bright region along the Galactic plane with $|l|<50^{\circ}$ and $|b|<10^{\circ}$ are within the GSC field of view. We find that the background count rate is significantly different from counter to counter. This is probably due to the difference in the configuration of the counter relative to the ISS and to its direction of orbital motion. Moreover, the background count rate is found to be highly variable on a time scale of days, dependent on the various observational conditions, such as the motion of the solar paddles and the shuttle docking to the ISS.

It is widely known that the short-term variation of the background is tightly correlated with cut-off rigidity (COR) for an X-ray instrument on a low-earth orbit (e.g., Hayashida et al. 1989; Tawa et al. 2008). We confirm such a trend also in the case of MAXI/GSC (Sugizaki et al. 2011), as shown in figure 2, which plots the GSC background count rate against COR of the ISS position. We model this background-COR relation by a third order polynomial, which is shown with the solid line in figure 2 .

Utilizing the MAXI simulator (Eguchi et al. 2009), we create background events for the individual counter, with a rate predicted by the background-COR correlation given in figure 2, after the long-term variation is considered. The detector coordinate DETX and the pulse height of each event are randomly assigned in the simulator ac- cording to the observed distributions of DETX and pulse height, both of which depend on COR (Sugizaki et al. 2011). The simulated event list is then processed in the same way as for the real data, and the sky position in R.A. and DEC. are assigned to each event by referring to the attitude of MAXI and the orbital motion of the ISS.

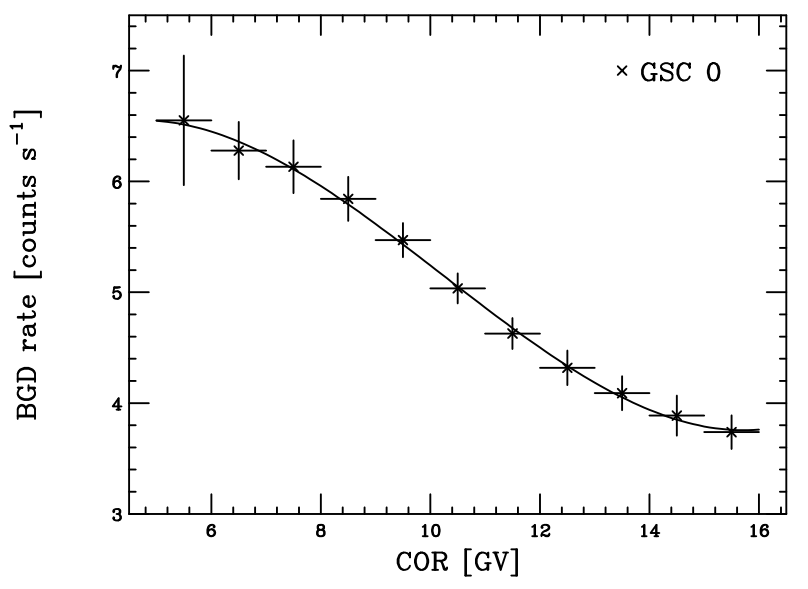

Fig. 2. An example of COR dependence of the GSC background count rate for the counter GSC_0. The peak of the COR-sorted daily-averaged background count rate distribution is plotted, together with its standard deviation.

\subsection{Image Analysis}

Source detection analysis is performed for a tangentially projected image of a small area in the "sky coordinates", as in the usual case of data analysis of pointing satellites. An image in the sky coordinates, $(X, Y)$, is defined by the reference point in R.A. and Dec. corresponding to the image center, the pixel size in units of degree in each direction, and the position angle between the $+Y$-axis and the north direction, which is set to be zero in our analysis. The entire sky is divided into 768 square images with a size of $14^{\circ} \times 14^{\circ}$ so that neighboring regions have enough overlapping area with a width of $>3$ degree in both sides, not to miss any sources near the boundary. The center positions of the images have equal spacing one another, which are adopted from those of the "HEALPix" ${ }^{1}$ grid (Górski et al. 2005). Each sky image is produced in the 4-10 keV band from the event list, referring to the columns of energy (PI) and sky position (R.A. and Dec. ). The bin width is set to be 0.1 degree, sufficiently finer than the point spread function of MAXI (Sugizaki et al. 2011).

\subsubsection{Step 1: Searching for Source Candidates}

For a divided region of the entire sky as noted above, we prepare the images of the real data and simulated background data. The background image is produced on the basis of the model described in section 3.1, by generating 10 times more photons than the actual rate to make the statistical errors negligibly small compared with those in the observed data. By subtracting the background from the data, we obtain the "residual" image. An example of

\footnotetext{
http://healpix.jpl.nasa.gov
} 
the real data is displayed in the left panel of figure 3 .

To find source candidates from statistical argument, the data and residual images are smoothed with a circle of $r=1^{\circ}$ with a constant weight of unity (i.e., simple integration). From the integrated counts of the data and residual, we calculate the excess significance at each point as "residual $/ \sqrt{\text { data" }}$, thus producing the "significance map" (right panel of figure 3). Then, we search for a peak showing the highest significance in the given image, which is listed as a source candidate if it exceeds $4 \sigma$. Next, we mask the circular region around its peak position with a radius of 3 degree, where the signals are expected to originate from the same source due to the point spread function. This step is repeated to find another source, which is added to the list of source candidates until the significance of the peak becomes lower than $4 \sigma$.

In this step, the normalization level of the background profile is estimated from the data themselves for each image, to absorb any remaining systematic errors in reproduction of the background rate, and also to approximately take into account contamination of Galactic diffuse emission in the background. To do this, we first detect bright sources above $15 \sigma$ from the significance map produced by assuming the nominal background rate predicted by the model. Then, after masking out circular regions of $r=3^{\circ}$ around these bright sources, we tune the normalization of the background level so that its total count matches that of the observed data in the same sky region. The tuned level could be slightly overestimated because sources with significances less than $15 \sigma$ are ignored in the first stage. This is not a problem, however, since we set a conservative threshold for picking up the source candidates $(4 \sigma)$ compared with that for the final catalog $(7 \sigma)$. Furthermore, as described later (section 3.2.2), we will make iteration of this process to ensure the completeness of the source search by adopting a more accurate background level.

After performing the source search in all the images, we merge the source candidate lists from all of them. In some cases, one source can be independently detected in multiple images, especially when it is located around the edge of the images. We regard any pairs of source candidates whose positional separation is smaller than 1.0 degree are the same object, and we only leave the source detected at the closer position to the image center in the merged list. Further, we also eliminate the source candidates which are regarded as obviously fake detections by visual inspection, such as those detected around a very bright source (like Sco X-1) or over strong extended diffuse emission from a galaxy cluster. We finally have 499 source candidates above $4 \sigma$ at $|b|>10^{\circ}$ in Step 1 .

\subsubsection{Step 2: Determining Flux and Position}

As preparation for the image fitting analysis, we construct the point spread function (PSF) model of each source candidate identified in Step 1. We assume that all sources can be treated as point-like in our data. One complexity in the analysis of the MAXI data is that the PSF depends on the position of the detector, and hence its integrated shape in the sky coordinates over multiple orbits is determined by the orbit and attitude condition of MAXI (Sugizaki et al. 2011). To take this into account, we utilize the MAXI simulator (Eguchi et al. 2009) to construct the PSF model in the sky image under the exactly same conditions as for real data. To suppress the statistical errors in the model, a sufficiently larger number of photons are generated by adopting the Crab Nebula flux (1 Crab) for all source candidates. In the simulation, we choose the same spectrum as the Crab Nebula ${ }^{2}$ : an absorbed power law with a photon index of 2.1 and a column density of $2.6 \times 10^{21} \mathrm{~cm}^{-2}$. We confirm that the choice of spectrum does not affect the flux determination, even though the PSF also has a weak energy dependence. Comparing the PSF with the data of the brightest point source Sco X-1, we find that the PSF model reproduces the data with residuals corresponding to $\sim 4 \%$ level of the peak height at $r \sim 1.0$ degree. These systematic errors in the PSF profile do not affect the flux determination of any sources in our catalog that can be regarded as point-like compared with the PSF size.

To determine the flux and position of the source candidates, we perform image fit to the real data in photon count space with a model consisting of the PSFs from the sources and the background. Only the inner region of $11^{\circ} \times 11^{\circ}$ is utilized to ignore the contribution from PSFs of sources located just outside the whole image region of $14^{\circ} \times 14^{\circ}$. To properly treat statistics with small numbers of photons in each bin, the Poisson maximum likelihood algorithm with MINUIT package (James \& Roos 1975) is adopted, based on the so-called $C$ statistics (Cash 1979), defined as

$$
C \equiv 2 \sum_{i, j}\{M(i, j)-D(i, j) \ln M(i, j)\},
$$

where $D(i, j)$ and $M(i, j)$ represent the data and model at the image pixel $(i, j)$, respectively. The best-fit parameters are obtained by minimizing the $C$ value, and the $1 \sigma$ statistical error of a single parameter can be estimated by finding that giving the $C$ value larger by unity than the best-fit. In the fitting process, the normalization of the PSF (i.e., flux) and its position are set to free parameters for all the source candidates, as well as the background level. Figure 4 shows the projection of the left panel of figure 3 onto X-axis (black), superposed with the best-fit model (red: total, blue: background). Since we treat all the sources as point-like, a few bright galaxy clusters may have a small uncertainty in the flux due to their extended structures significantly larger than the PSF (see Note in table 1).

We define the detection significance $\left(s_{\mathrm{D}}\right)$ of each source as

$$
s_{\mathrm{D}} \equiv \text { (best-fit flux)/(its } 1 \sigma \text { statistical error). }
$$

In this paper, we adopt $s_{\mathrm{D}}>7$ as the detection criteria for the final catalog, which is conservative enough not to contain any fake detections within current systematic errors in the background model. We verify this by checking the

\footnotetext{
2 The spectral parameters are adopted from the INTEGRAL General Reference Catalog (ver. 31). http://www.isdc.unige.ch/integral/science/catalogue
} 

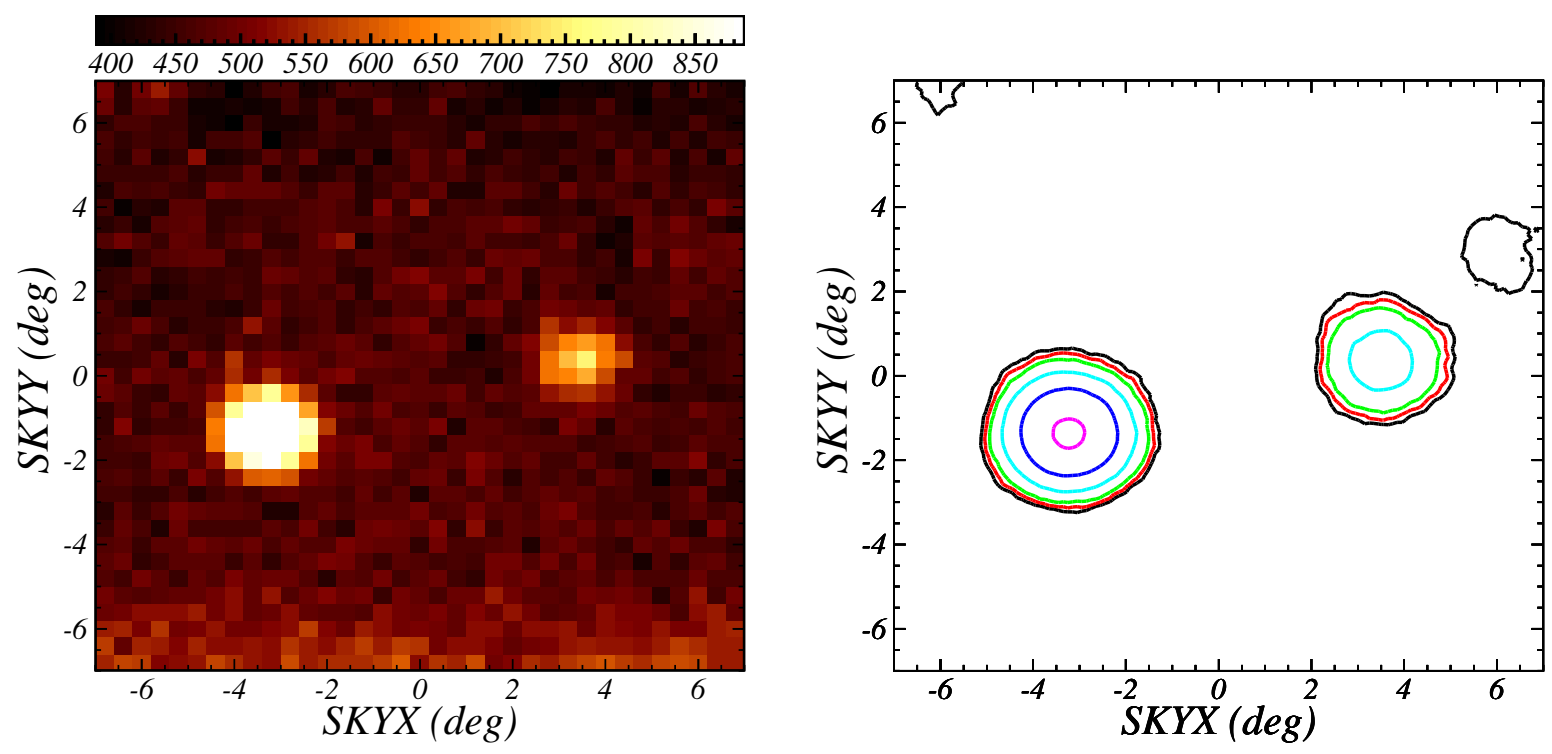

Fig. 3. (Left) An example of observed image in photon count space. (Right) The significance map of the same region. The black, red, green, cyan, blue, and purple contours represent the levels of significance of 5, 7, 10, 20, 40, and 70, respectively.

reproductivity of the background profile on a scale of the PSF size $\left(\sim 3^{\circ}\right)$ by the following analysis. We search for "negative" signals in the residual image by changing the sign of the significance map in Step 1. Then, we perform image fitting with a model consisting of the background plus negative PSFs identified in the previous step. As a result, it is confirmed that no negative peaks are detected with significances above $7 \sigma$ from all the images. This indicates that the number of fake, positive peaks is also expected to be zero.

Using the tentative source list produced in this way, we repeat the same procedure of Step 1 by applying the bestfit "background plus PSFs" model determined in Step 2, instead of the pure background model as done in the first analysis. The main purpose is to search for missing sources located close to a nearby, brighter source because of the mask around the peak with a radius of 3 degree applied to the significance map in Step 1. Finally, we perform image fitting by adding these newly identified source candidates into the model. The final number of the detected sources with $s_{\mathrm{D}}>7$ is 143 at $|b|>10^{\circ}$, including additional 4 sources found by this iteration process.

The solar paddle structures on the ISS sometimes block a part of the field of view of MAXI, which works to reduce the apparent averaged count rate of an X-ray source. Since we do not take into account these effects in the image analysis, we must evaluate the shielding effect to derive the correct fluxes of the cataloged sources. To estimate it, we perform the whole sky simulations of the CXB with and without the solar paddle occultation during the 7month period. By comparing these results, we estimate the fraction of unocculted observing time at each sky position. It is found to be $\approx 96 \%$ on average and in a range of 90\%-100\%, depending on the position. Thus, we correct

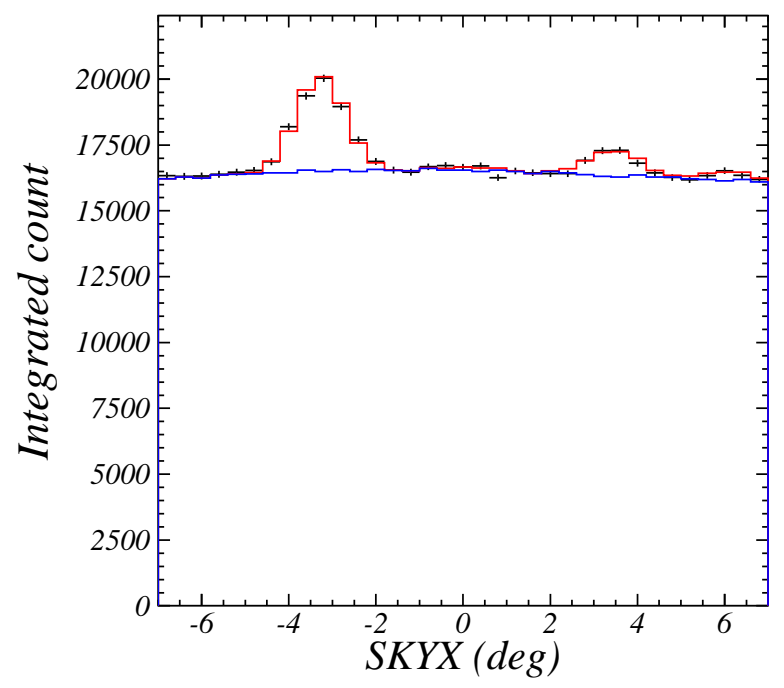

Fig. 4. Comparison of the observed data (black) and the best-fit model (red: total, blue: only background). The faintest source in this image is located at $X=6.0$ with $s_{\mathrm{D}} \approx 7$.

the flux obtained in Step 2 for this fraction according to the sky position, which is finally listed in the catalog.

\section{MAXI CATALOG}

\subsection{X-Ray Properties}

In table 1, we present the first MAXI/GSC catalog at high Galactic latitudes $\left(|b|>10^{\circ}\right)$ compiled from the 7month data. It contains 143 sources detected with signifi- 
cance above $7 s_{\mathrm{D}}$ in the $4-10 \mathrm{keV}$ band, sorted by R.A. and Dec. . The 1st to 7th columns give (1) source identification number, (2) MAXI source name designated from the detected position, $(3,4)$ MAXI best-fit position in R.A. and Dec., (5) detection significance $s_{\mathrm{D}},(6)$ flux in the $4-$ $10 \mathrm{keV}$ band converted from the count rate by assuming a photon index of 2.1 , and (7) its $1 \sigma$ statistical error.

The left panel of figure 5 shows the correlation between the detection significance $\left(s_{\mathrm{D}}\right)$ and the flux. It is seen that $s_{\mathrm{D}}$ is roughly proportional to the flux in the low flux region where the background determines the noise (statistical fluctuation of photon counts). Whereas in the high flux region, it is proportional to (flux $)^{1 / 2}$ since the source photons dominate the noise. The scatter is mainly due to the variation of exposure (see figure 1). The histograms of flux and $s_{\mathrm{D}}$ are shown in the middle and right panels of figure 5, respectively. The limiting sensitivity is found to be $\sim 1.2 \mathrm{mCrab}$ with $s_{\mathrm{D}}>7$, corresponding to $\sim 1.5 \times 10^{-11}$ ergs $\mathrm{cm}^{-2} \mathrm{~s}^{-1}$ in the $4-10 \mathrm{keV}$ band. This is fully consistent with our expectation from the actual background level and observing efficiency of MAXI (Ueda et al. 2010).

\subsection{Identification}

Source identification is one of the most important task for the catalog obtained from an unbiased survey. Since the position error of MAXI is typically $0.4^{\circ}$ for sources with $s_{\mathrm{D}}=7$ at $90 \%$ confidence level (section 4.3 ), direct identification in the optical or near infrared band solely based on the position is practically difficult without further localization. Therefore, we cross correlate our catalog with major X-ray/gamma-ray catalogs for which optical identification or position determination with an $\sim$ arcsec accuracy is available, assuming that the MAXI sources are likely detected also in these catalogs with similar flux limits. Table 2 lists the catalogs and references we use for the cross correlation; Swift/BAT 58-month and 54-month Catalogs, 1st Fermi/LAT AGN Catalog, the brightest 2200 sources in the $0.5-2 \mathrm{keV}$ band in the RASS BSC, INTEGRAL General Reference Catalog (version 31), NORAS Galaxy Cluster Survey Catalog, REFLEX Galaxy Cluster Survey Catalog, and 1st XMM-Newton Slew Survey Catalog.

To cross correlate the MAXI/GSC catalog with the reference ones, we only take into account the position error of MAXI, $\sigma_{\text {pos }}$, since those in the other catalogs are negligibly small. It is defined as

$$
\sigma_{\mathrm{pos}} \equiv \sqrt{\sigma_{\text {stat }}^{2}+\sigma_{\mathrm{sys}}^{2}}
$$

where $\sigma_{\text {sys }}$ is the systematic error of 0.05 degree $(1 \sigma$, section 4.3) and $\sigma_{\text {stat }}$ is the statistical one combined from those in the $X$ and $Y$ directions at $1 \sigma$ obtained in the image fit process (Step 2). To be conservative, we adopt $3 \sigma_{\text {pos }}$ as the radius for the position matching. We find that this roughly corresponds to a confidence level of $99 \%$ in the 2-dimensional space, giving a typical increment in the $C$ value by 9.2 .

The total numbers of possible counterparts found from each reference catalog are also summarized in table 2 .
We identify the MAXI sources largely on the basis of this cross-correlation results. Basically, we first look for counterparts in the Swift/BAT and INTEGRAL catalogs, which achieve similar sensitivities to that of MAXI for sources without heavy obscuration. If no sources are found from these catalogs, we also refer to the results with the other catalogs. Ten MAXI sources are found to have no matched counterparts in any of these catalogs within $3 \sigma_{\text {pos }}$. In such cases, we identify the counterpart by individual inspection using $\mathrm{NED}^{3}$ and $\mathrm{SIMBAD}^{4}$.

To estimate the number of possible spurious identification, we calculate the expected number of coincidental matches between the MAXI sources and those in the reference catalogs, $N_{\mathrm{cm}}$, using the following equation:

$$
N_{\mathrm{cm}}=\sum_{i} \rho_{i} \times S_{i},
$$

where the suffix $i$ denotes each reference catalog that can be considered to be independent, and $\rho_{i}$ and $S_{i}$ are the mean surface number-density of sources in the reference catalog and the total area within the $3 \sigma_{\text {pos }}$ error radii of MAXI sources searched for their counterparts in that catalog, respectively. Here we assume that the spatial distribution of sources in each catalog can be regarded to be random, which is a good approximation in the high Galactic-latitude sky. For simplicity, we only refer to the Swift/BAT 58-month Catalog and the RASS BSC for this calculation, which cover the different energy bands and hence are complementary each other. Following our actual identification procedure, we first consider the Swift/BAT 58-month Catalog and the error region of all MAXI sources, which correspond to $\rho_{1}=0.022 \mathrm{deg}^{-2}$ and $S_{1}=42 \mathrm{deg}^{2}$, respectively. Then, to reflect the fact that the remaining MAXI sources without hard X-ray counterparts have been mainly identified by the RASS catalog, we adopt $\rho_{2}=0.054 \mathrm{deg}^{-2}$ for the 1829 brightest RASS sources and $S_{2}=18 \mathrm{deg}^{2}$ for the MAXI error regions. In this way, we obtain $N_{\mathrm{cm}}=1.9$. Note that this is a conservative estimate since we have neglected the duplicative sources between the Swift/BAT and RASS catalogs in the calculation.

Out of the total 143 MAXI sources, we finally identify 142 sources, and only one source ${ }^{5}$ remains unidentified. The locations of the cataloged sources are plotted in figure 6 in the Galactic coordinates with different colors corresponding to different types of object. Table 3 gives a summary of source identification. The catalog contains 38 Galactic/LMC/SMC objects, 48 galaxy clusters, 51 AGNs including 12 blazars. The detailed information of the counterparts is provided in the 8 th to 14 th columns of table 1 for each MAXI source, (8) the source name, (9, 10) the position of the counterpart in R.A. and Dec., (11) type, (12) redshift (only for extragalactic objects), (13) other names of the source, and (14) notes in special cases. We find four MAXI sources ${ }^{6}$ are probably confused from

\footnotetext{
http://ned.ipac.caltech.edu

http://simbad.u-strasbg.fr/simbad

MAXI J0457-696

MAXI J0627-540, J0957+693, J1633-750, and J1941-104
} 

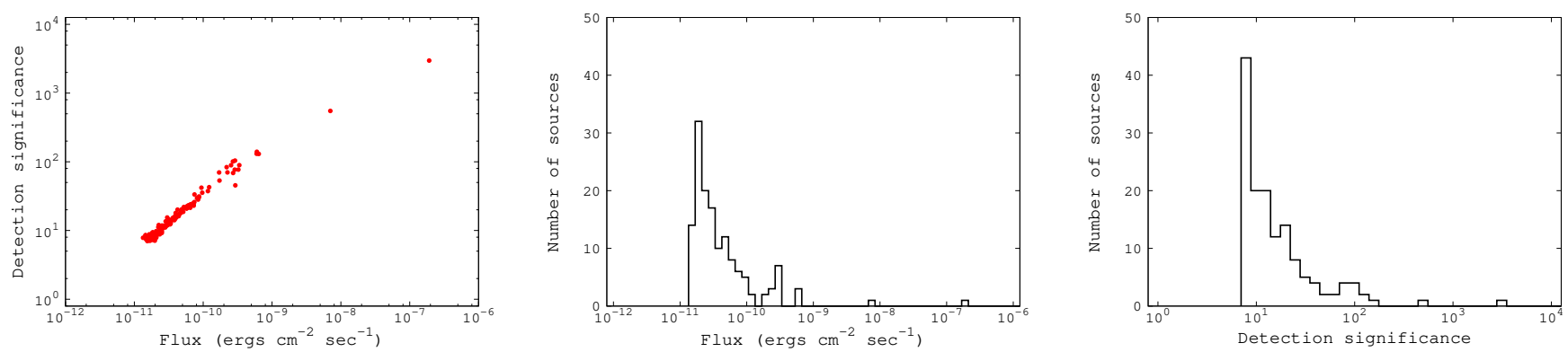

Fig. 5. The correlation between the detection significance and the flux for all the sources in the MAXI/GSC catalog (left), the distribution of the flux (middle), and that of the detection significance (right).

Table 2. Reference catalogs used for cross correlation.

\begin{tabular}{lcl}
\hline \hline Catalog & $\begin{array}{c}\text { Number of } \\
\text { matched sources }\end{array}$ & Reference \\
\hline Swift/BAT 58-month Catalog & 93 & Baumgartner et al. 2010* \\
54-month Palermo BAT-Survey Catalog (2PBC) & 100 & Cusumano et al. 2010 \\
1st Fermi/LAT AGN Catalog (1LAC) & 12 & Abdo et al. 2010 \\
RASS Bright Souce Catalog (1RXS) & 110 & Voges et al. 1999 \\
INTEGRAL General Reference Catalog (ver. 31) & 108 & INTEGRAL Science Data Centre website ${ }^{\dagger}$ \\
NORAS Galaxy Cluster Survey Catalog & 14 & Böhringer et al. 2000 \\
REFLEX Galaxy Cluster Survey Catalog & 17 & Böhringer et al. 2004 \\
1st XMM-Newton Slew Survey Catalog (XMMSL1) & 65 & Saxton et al. 2008 \\
* The data is retrieved from NASA website: http://heasarc.nasa.gov/docs/swift/results/bs58mon \\
${ }^{\dagger}$ http://www.isdc.unige.ch/integral/science/catalogue
\end{tabular}

multiple objects that are difficult to be resolved with the angular resolution of MAXI. For those sources, multiple counterparts are listed in table 1 , which are not counted in the identification summary shown in table 3.

We compare the number statistics of source populations in the first MAXI/GSC catalog with those in the HEAO-1 A-2 $\left(|b|>20^{\circ}\right.$, Piccinotti et al. 1982), RXTE $\left(|b|>10^{\circ}\right.$, Revnivtsev et al. 2004), and Swift/BAT (Cusumano et al. 2010) catalogs. The number ratios between Galactic objects, AGNs, and galaxy clusters in our catalog, 21:40:36 $\left(|b|>20^{\circ}\right)$ and $38: 51: 48\left(|b|>10^{\circ}\right)$, are found to be consistent with both HEAO-1 A-2 (17:29:30) and RXTE (63:100:64) results within the statistical errors. By contrast, the Swift/BAT survey performed in the 15-150 keV band brings a significantly higher fraction of AGNs at $|b|>10^{\circ}, \sim 60 \%$, than these catalogs produced in lower energy bands. We confirm that all but two galaxy clusters in the HEAO-1 A-2 catalog are listed in ours, with the remaining two also detected with slightly lower significances than $s_{\mathrm{D}}=7$ in the MAXI/GSC data. We note, however, that about $40 \%(=12 / 29)$ of the Piccinotti AGNs are absent in our catalog. In fact, the X-ray fluxes of these 12 AGNs obtained from pointing observations with ASCA, XMM-Newton, or Swift/XRT in more recent years (Winter et al. 2009, Shinozaki et al. 2006, Aharonian et al. 2005, and Blustin et al. 2004) are all lower than the typical limiting sensitivity of the MAXI survey, $\approx 2 \times 10^{-11}$
Table 3. Categories of cataloged sources.

\begin{tabular}{cc}
\hline \hline Category & Number of sources \\
\hline unidentified & 1 \\
galaxies & 1 \\
galaxy clusters & 48 \\
Seyfert galaxies & 39 \\
blazars & 12 \\
CVs/Stars & 20 \\
X-ray binaries & 18 \\
confused & 4 \\
\hline
\end{tabular}

ergs $\mathrm{cm}^{-2} \mathrm{~s}^{-1}$ in the $4-10 \mathrm{keV}$ band (figure 9, in section 4.4), by assuming a power law photon index of 2 . This fact indicates that the list of the brightest X-ray AGNs in the whole sky has significantly changed since $\sim 30$ years ago due to their long-term variability.

\subsection{Position Accuracy}

Utilizing the source identification result, we estimate the positional error of MAXI as a function of the detection significance, which is observationally determined. Therefore, it gives the most robust information useful for identification work of MAXI sources within the current calibration. Figure 7 shows the source distributions as a 


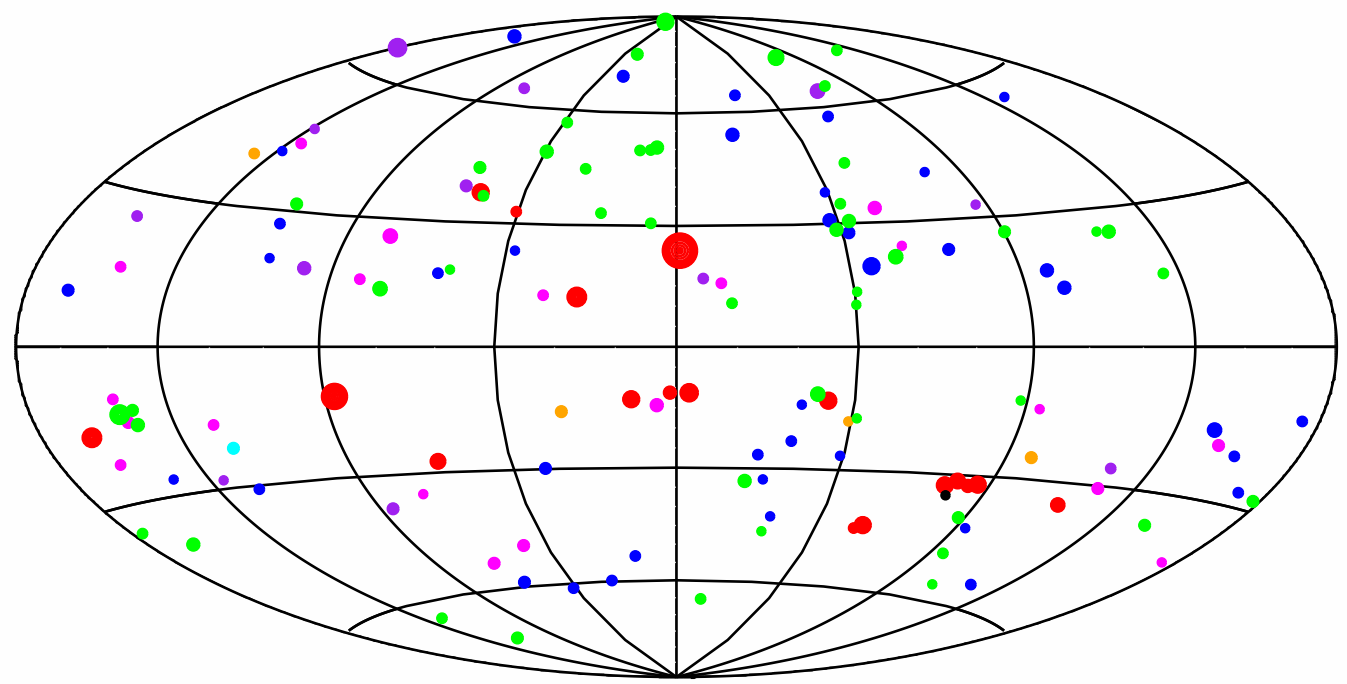

Fig. 6. Locations of cataloged sources with $s_{\mathrm{D}}>7$ in the Galactic coordinates. The radius is proportional to logarithm of the 4-10 keV flux. Different colors correspond to different types of sources: unidentified (black); galaxies (cyan); galaxy clusters (green); Seyfert galaxies (blue); blazars (purple); CVs/Stars (magenta); X-ray binaries (red); and confused (orange).

function of the angular separation between the MAXI position and that of the counterpart in different $s_{\mathrm{D}}$ ranges; $s_{\mathrm{D}}=7-10$ (left), $s_{\mathrm{D}}=10-30$ (middle), and $s_{\mathrm{D}}>30$ (right). From each distribution, we measure a $90 \%$ error radius by counting the number of sources.

Figure 8 plots the $90 \%$ error radius as a function of the detection significance. In the highest $s_{\mathrm{D}}$ bin, the statistical errors are expected to be negligibly small compared with the systematic errors due to those in the attitude determination and position calibration of the GSC (Sugizaki et al. 2011). We thus estimate the systematic error to be $\sigma_{\text {sys }}^{90} \sim 0.08$ degree $(90 \%$ confidence level $)$, or $\sigma_{\text {sys }} \sim 0.05$ degree $(1 \sigma)$. In the lower significance region, the total error is dominated by the statistical error, which is expected to be proportional to $s_{\mathrm{D}}^{-1}$. We obtain the best-fit formula for the $90 \%$ positional error of MAXI sources

$$
\sigma_{\text {pos }}^{90}\left(s_{\mathrm{D}}\right)=\sqrt{\left(A / s_{\mathrm{D}}\right)^{2}+\left(\sigma_{\text {sys }}^{90}\right)^{2}},
$$

where $A=3.08 \pm 0.04$ and $\sigma_{\text {sys }}^{90}=0.08$.

\section{4. $\log N-\log S$ Relation}

Source number counts (log $N-\log S$ relation) give the most fundamental statistical properties of source populations that can be derived from survey observations. To obtain this, we need to have an area curve, where survey area guaranteed for detection of a source with the given detection criteria (i.e., $s_{\mathrm{D}}>7$ ) is given as a function of flux. We estimate the sensitivities at each sky position from (1) the background photon counts and (2) the product of effective detector area $\times$ exposure obtained from the CXB simulation (shown in figure 1). We confirm that the actual detection significance derived from the PSF fit can be well approximated by that analytically calculated

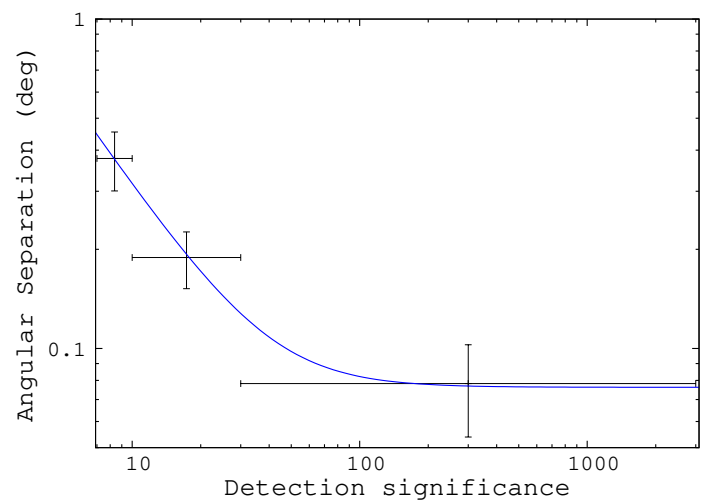

Fig. 8. 90\% error radius of MAXI sources plotted as a function of the detection significance. The blue curve indicates the best-fit formula with the form of $\sqrt{\left(3.08 / s_{\mathrm{D}}\right)^{2}+(0.08)^{2}}$ (degree).

from these parameters on the basis of a simple statistical argument. Figure 9 plots the area curve calculated in this way; we confirm that the $7 \sigma$ sensitivity is $\approx 1.2 \mathrm{mCrab}$, or $1.5 \times 10^{-11}$ ergs $\mathrm{cm}^{-2} \mathrm{~s}^{-1}$, in the $4-10 \mathrm{keV}$ band.

Dividing the flux distribution of the detected sources by the survey area gives $\log N-\log S$ relation in the differential form. Figure 10 shows that in the integral form, where the source number density $N$ above flux $S$ is plotted. We separately plot those of all the sources and extragalactic objects. The results for extragalactic objects are in an excellent agreement with the HEAO-1 A-2 result by Piccinotti et al. 1982, by converting the fluxes from the 2-10 keV band into the $4-10 \mathrm{keV}$ band assuming a photon 

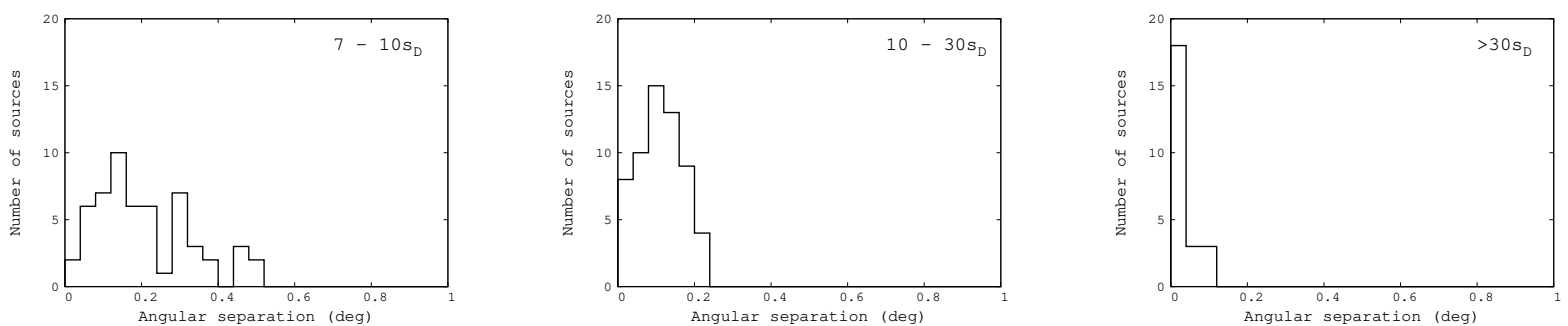

Fig. 7. Source distributions as a function of the angular separation between the MAXI position and that of the counterpart for different regions of detection significance (from left to right, $7-10 s_{\mathrm{D}}, 10-30 s_{\mathrm{D}}$, and $>30 s_{\mathrm{D}}$ ).

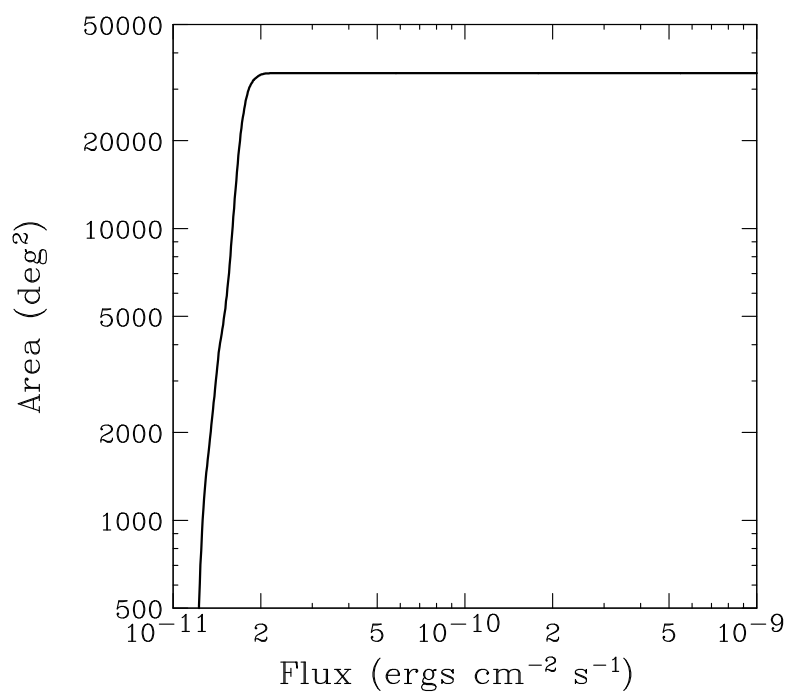

Fig. 9. Area curve of the 7-month MAXI/GSC survey at $|b|>10^{\circ}$ in the $4-10 \mathrm{keV}$ band, for the detection significance of $s_{\mathrm{D}}>7$.

index of 2. We recall, however, that the actual content of the AGN sample has been significantly changed since the HEAO-1 A2 era, as mentioned in section 4.2.

\section{CONCLUSION}

We present the first MAXI/GSC catalog produced from an unbiased X-ray survey in the $4-10 \mathrm{keV}$ band at high Galactic latitude sky $\left(|b|>10^{\circ}\right)$. The initial 7 -month data after the start of normal operation are utilized here. The limiting sensitivity of $1.5 \times 10^{-11} \mathrm{ergs} \mathrm{cm}^{-2} \mathrm{~s}^{-1}(1.2$ $\mathrm{mCrab})$ is achieved for the detection significance criteria of $>7 s_{\mathrm{D}}$, which is conservatively adopted in this paper not to contain fake sources under the current calibration. The sensitivity already exceeds that of the HEAO-1 A-2 all-sky survey in the $2-10 \mathrm{keV}$ band. It is expected to be improved significantly by adding more data and by further improvement of the background model calibration.

The catalog contains 143 sources, which are identified as 38 Galactic/LMC/SMC objects, 48 galaxy clusters, 39 Seyferts, 12 blazars, 1 galaxy, and 4 confused objects. Only one source remains unidentified. The high completeness of this catalog makes it particularly useful to investi-

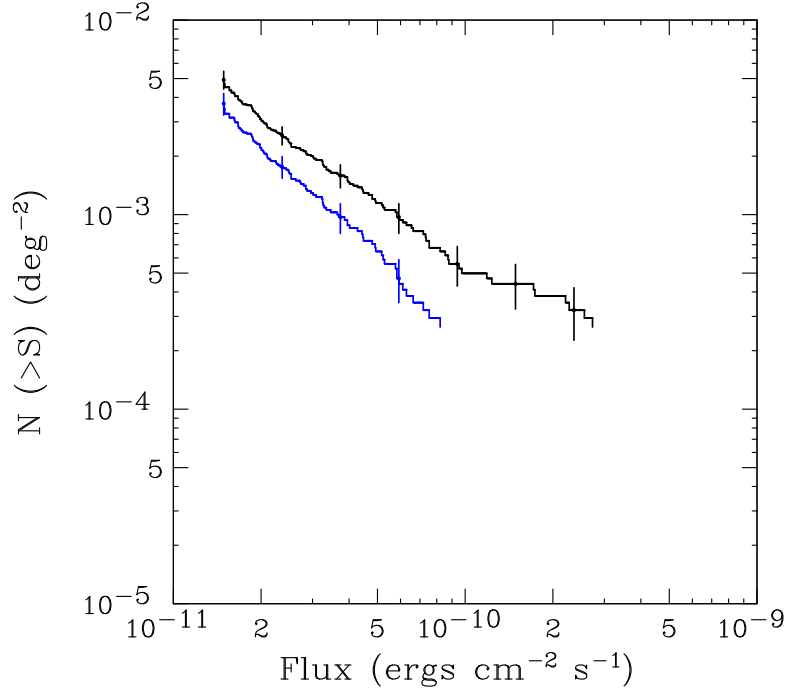

Fig. 10. $\log N-\log S$ relations in the $4-10 \mathrm{keV}$ band obtained from the 7-month MAXI/GSC survey at $|b|>10^{\circ}$. From top to bottom, those of the total sources (black) and extragalactic objects (blue). The error bars correspond to the $90 \%$ statistical errors in the source counts.

gate the statistical properties of X-ray populations in the local universe with the least uncertainties. The initial results on the X-ray luminosity function of Seyfert galaxies are reported in the accompanying paper.

This research has made use of the NASA/IPAC Extragalactic Database (NED) which is operated by the Jet Propulsion Laboratory, California Institute of Technology, under contract with the National Aeronautics and Space Administration. This research has made use of the SIMBAD database, operated at CDS, Strasbourg, France. Some of the results in this paper have been derived using the HEALPix (K.M. Górski et al., 2005, ApJ, 622, p759) package. This research was partially supported by the Ministry of Education, Culture, Sports, Science and Technology (MEXT), Grant-in-Aid No.19047001, 20041008, 20244015 , 20540237, 21340043, 21740140, 22740120, 23000004, 23540265, and GlobalCOE from MEXT "The Next Generation of Physics, Spun from Universality and Emergence" and "Nanoscience and Quantum Physics". 


\section{References}

Abdo, A. A., et al. 2010, ApJ, 715, 429

Aharonian, F., et al. 2005, A\&A, 442, 895

Baumgartner, W. H., et al. 2010, ApJS, submitted

Beckmann, V., Gehrels, N., Shrader, C. R., \& Soldi, S. 2006, ApJ, 638, 642

Beckmann, V., et al. 2009, A\&A, 505, 417

Bird, A. J., et al. 2007, ApJS, 170, 175

Bird, A. J., et al. 2010, ApJS, 186, 1

Blustin, A. J., Page, M. J., \& Branduardi-Raymont, G. 2004, A\&A, 417, 61

Böhringer, H., et al. 2000, ApJS, 129, 435

Böhringer, H., et al. 2004, A\&A, 425, 367

Cash, W. 1979, ApJ, 228, 939

Cusumano, G., et al. 2010, A\&A, 524, A64

Eguchi, S., Hiroi, K., Ueda, Y., Sugizaki, M., Tomida, H., Suzuki, M., \& The Maxi Team 2009, Astrophysics with All-Sky X-Ray Observations, 44

Górski, K. M., Hivon, E., Banday, A. J., Wandelt, B. D., Hansen, F. K., Reinecke, M., \& Bartelmann, M. 2005, ApJ, 622,759

Guainazzi, M., Piconcelli, E., Jiménez-Bailón, E., \& Matt, G. 2005, A\&A, 429, L9

Hayashida, K., Inoue, H., Koyama, K., Awaki, H., Takano, S., 1989, PASJ, 41, 373

Isobe, N., et al. 2010, PASJ, 62, L55

James, F., \& Roos, M. 1975, Computer Physics Communications, 10, 343

Krivonos, R., Revnivtsev, M., Lutovinov, A., Sazonov, S., Churazov, E., \& Sunyaev, R. 2007, A\&A, 475, 775

Matsumura, T., et al. 2011, The Astronomer's Telegram, 3308, 1

Matsuoka, M., et al. 2009, PASJ, 61, 999

Mihara, T., et al. 2011, PASJ, in press

Piccinotti, G., Mushotzky, R. F., Boldt, E. A., Holt, S. S., Marshall, F. E., Serlemitsos, P. J., \& Shafer, R. A. 1982, ApJ, 253, 485

Revnivtsev, M., Sazonov, S., Jahoda, K., \& Gilfanov, M. 2004, A\&A, 418, 927

Saxton, R. D., Read, A. M., Esquej, P., Freyberg, M. J., Altieri, B., \& Bermejo, D. 2008, A\&A, 480, 611

Schwope, A., et al. 2000, Astronomische Nachrichten, 321, 1

Sekiguchi, K., \& Wolstencroft, R. D. 1992, MNRAS, 255, 581

Shinozaki, K., Miyaji, T., Ishisaki, Y., Ueda, Y., \& Ogasaka, Y. 2006, AJ, 131, 2843

Sugizaki, M., et al. 2011, PASJ, in press

Suzuki, M., et al. 2010, The Astronomer's Telegram, 2401, 1

Tawa, N., et al., 2008, PASJ, 60, S11

Tomida, H., et al. 2011, arXiv:1101.3651

Tsunemi, H., Tomida, H., Katayama, H., Kimura, M., Daikyuji, A., Miyaguchi, K., Maeda, K., \& MAXI Team 2010, PASJ, 62, 1371

Tueller, J., Mushotzky, R. F., Barthelmy, S., Cannizzo, J. K., Gehrels, N., Markwardt, C. B., Skinner, G. K., \& Winter, L. M. 2008, ApJ, 681, 113

Tueller, J., et al. 2010, ApJS, 186, 378

Ueda, Y., Hiroi, K., Isobe, N., Hayashida, M., Sugizaki, M., \& Maxi Team 2010, The First Year of MAXI: Monitoring Variable X-ray Sources, 4th International MAXI Workshop held November 30 - December 2, 2010 in Tokyo, Japan

Voges, W., et al. 1999, A\&A, 349, 389

Voges, W., et al. 2000, IAU Circ., 7432, 3
Winter, L. M., Mushotzky, R. F., Reynolds, C. S., \& Tueller, J. 2009, ApJ, 690, 1322 


\section{arXiv:1108.5516v1 [astro-ph.HE] 29 Aug 2011}

Table 1. Cataloged sources in the MAXI/GSC survey.

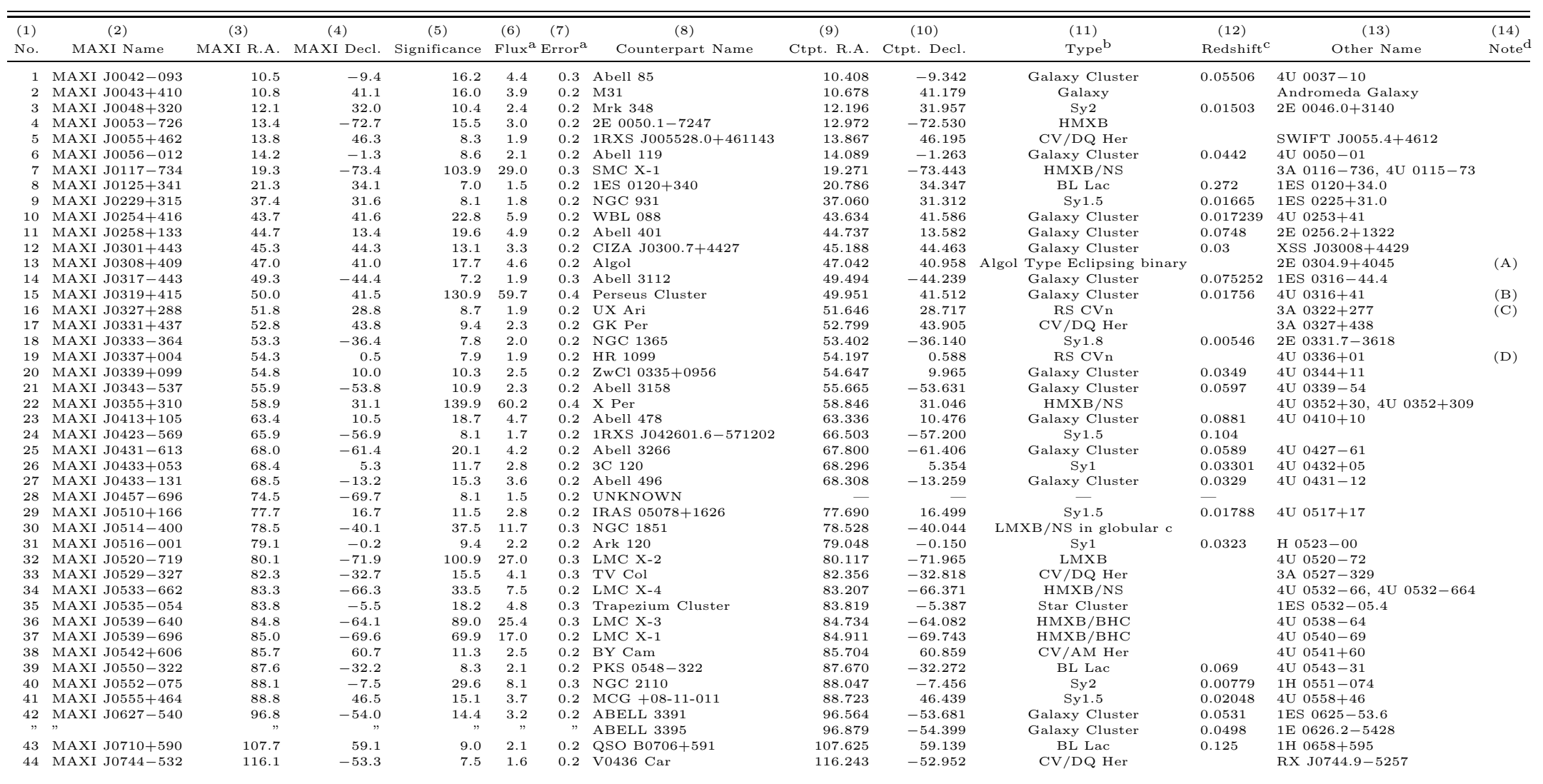


Table 1 (cont'd)

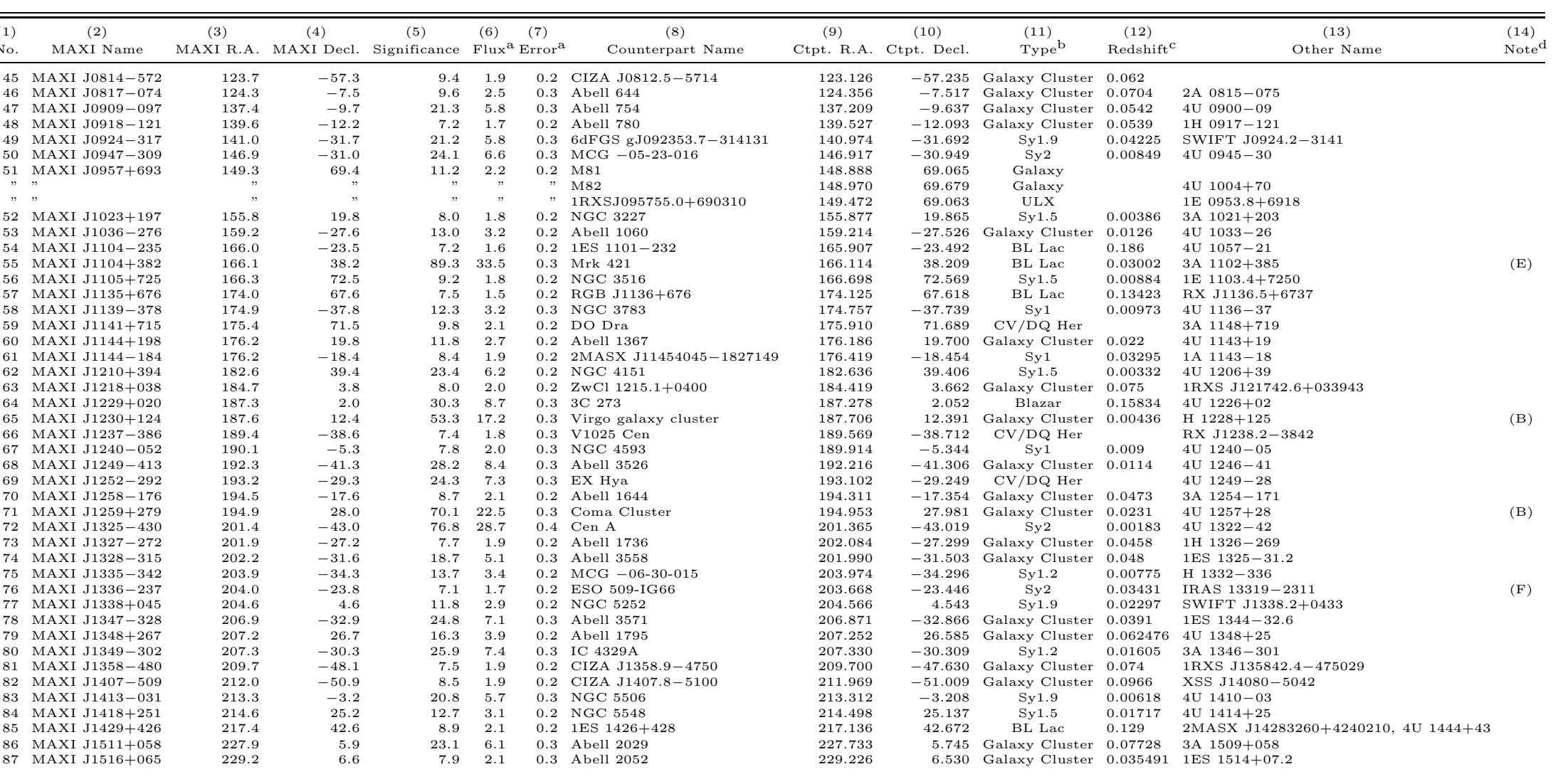


Table 1 (cont'd)

\begin{tabular}{|c|c|c|c|c|c|c|c|c|c|c|c|c|c|}
\hline $\begin{array}{l}\text { (1) } \\
\text { No. }\end{array}$ & $\begin{array}{c}(2) \\
\text { MAXI Name }\end{array}$ & $\begin{array}{c}\text { (3) } \\
\text { MAXI R.A. }\end{array}$ & $\begin{array}{c}(4) \\
\text { MAXI Decl. }\end{array}$ & $\begin{array}{c}\text { (5) } \\
\text { Significance }\end{array}$ & $\begin{array}{l}(6) \\
\text { Flux }^{\mathrm{a}}\end{array}$ & $\begin{array}{c}(7) \\
\text { Error }^{\mathrm{a}}\end{array}$ & $\begin{array}{c}(8) \\
\text { Counterpart Name }\end{array}$ & $\begin{array}{c}(9) \\
\text { Ctpt. R.A. }\end{array}$ & $\begin{array}{c}(10) \\
\text { Ctpt. Decl. }\end{array}$ & $\begin{array}{l}(11) \\
\text { Type }^{b}\end{array}$ & $\begin{array}{c}(12) \\
\text { Redshift }^{\mathrm{c}}\end{array}$ & $\begin{array}{c}(13) \\
\text { Other Name }\end{array}$ & $\begin{array}{l}(14) \\
\text { Note }^{d}\end{array}$ \\
\hline 88 & MAXI J1522+279 & 230.7 & 28.0 & 8.4 & 2.0 & 0.2 & Abell 2065 & 230.678 & 27.723 & alaxy Cluster & 0.0726 & $4 \mathrm{U} 1521+28$ & \\
\hline 89 & MAXI J1522+084 & $230.7 \mathrm{~F}>\mathrm{C}$ & 8.4 & 8.4 & 2.0 & 0.2 & Abell 2063 & 230.772 & 8,602 & alaxy Cluster & 0.03616 & $1 \mathrm{E}$ & \\
\hline 90 & MAXI J1558+272 & 239.7 & 27.3 & 21.2 & 5.2 & 0.2 & Abell 2142 & 239.567 & 27.225 & $\begin{array}{l}\text { Galaxy Cluster } \\
\text { Galust }\end{array}$ & $\begin{array}{ll}0.0909 \\
0\end{array}$ & $4 \mathrm{U}$ 1556-27 & \\
\hline 91 & MAXI J1602+160 & 240.6 & 16.0 & 10.9 & 2.6 & 0.2 & Abell 2147 & 240.572 & 15.895 & Galaxy Cluster & 0.035 & $4 \mathrm{U} 1601+15$ & \\
\hline 92 & MAXI J1603-759 & 240.8 & -75.9 & 8.5 & 1.5 & 0.2 & CIZA J1601.7-7544 & 240.445 & -75.746 & Galaxy Cluster & 0.153 & XSS J16019-7548 & \\
\hline 93 & MAXI J1615-058 & 243.9 & -5.8 & 8.8 & 2.3 & 0.3 & Abell 2163 & 243.946 & -6.146 & Galaxy Cluster & 0.1698 & $1 \mathrm{H} 1613-060$ & \\
\hline 94 & MAXI J1617-283 & 244.5 & -28.4 & 9.0 & 2.2 & 0.2 & 1RXS J161933.6-280736 & 244.887 & -28.127 & Symb/NS & & SWIFT J1619.4-2808 & (G) \\
\hline 95 & MAXI J1619-156 & 245.0 & -15.7 & 2976.9 & 19216.8 & 6.2 & & 244.980 & -15.640 & $\mathrm{LMXB} / \mathrm{NS}$ & & $4 \mathrm{U} 1617-15$ & \\
\hline 96 & MAXI J1626-335 & 246.7 & -33.5 & 9.9 & 2.5 & 0.2 & CIZA J1626.3-3329 & 246.586 & -33.489 & Galaxy Cluster & 0.1098 & XSS J16265-3303 & \\
\hline & & 246.8 & & 11.1 & & & 3EG J1627-2419 & 247.000 & & & & & \\
\hline 98 & MAXI J1628+396 & $247.2>>2>3$ & 39.6 & 16.9 & 4.4 & 0.2 & Abell 2199 & 247.154 & 39.524 & Galaxy Cluster & 0.030151 & $4 \mathrm{U} 1627+39$ & \\
\hline 99 & MAXI J1632-674 & 248.0 & -67.4 & 83.5 & 21.9 & 0.3 & $\mathrm{KZ} \operatorname{TrA}$ & 248.070 & -67.462 & LMXB & & $4 \mathrm{U} 1626-67,4 \mathrm{U} 1626-673$ & \\
\hline 100 & MAXI J1633+056 & 248.3 & 5.6 & 8.1 & 2.0 & 0.2 & Abell 2204 & 248.194 & 5.571 & Galaxy Cluster & 0.152158 & $2 \mathrm{E} 1630.3+0540$ & \\
\hline 101 & MAXI J1633-750 & 248.3 & -75.0 & 7.8 & 1.3 & 0.2 & ABELL 3628 & 247.755 & -75.176 & Galaxy Cluster & 0.1492 & XSS J16345-7506 & \\
\hline & & & & & & & 1RXS J163153.5-750631 & 247.973 & -75.109 & X-ray source & & & \\
\hline 102 & MAXI J1638-643 & 249.6 & -64.3 & 41.9 & 9.4 & 0.2 & TrA Cluster & 249.567 & -64.347 & Galaxy Cluster & 0.0508 & XSS J16384-6424 & \\
\hline 103 & MAXI J1653+398 & 253.4 & 39.8 & 13.2 & 3.2 & 0.2 & Mrk 501 & 253.468 & 39.760 & BL Lac & 0.03366 & $4 \mathrm{U} 1651+39$ & \\
\hline 104 & MAXI J1657+353 & 254.5 & 35.4 & 45.4 & 29.3 & 0.4 & Her X-1 & 254.458 & 35.342 & LMXB/NS & & $4 \mathrm{U} 1656+35$ & \\
\hline 105 & $\begin{array}{l}\text { MAXI J1701+341 } \\
\text { MAXI J1703+785 }\end{array}$ & $\begin{aligned} 255.3 \\
256.0\end{aligned}$ & 34.2 & $\begin{array}{r}9.2 \\
180\end{array}$ & 2.5 & 0.3 & Abell 2244 & 255.679 & 34.062 & Galaxy Cluster & 0.0968 & 3A $1702+340$ & \\
\hline $\begin{array}{l}106 \\
107\end{array}$ & $\begin{array}{l}\text { MAXI J1703+785 } \\
\text { MAXI J1706+241 }\end{array}$ & $\begin{array}{l}256.0 \\
256.7\end{array}$ & $\begin{array}{l}78.6 \\
24.1\end{array}$ & $\begin{array}{l}18.0 \\
10.0\end{array}-\longrightarrow$ & $\begin{array}{l}4.0 \\
2.3\end{array}$ & $\begin{array}{l}0.2 \\
0.2\end{array}$ & $\begin{array}{l}\text { Abell } 2256 \\
\text { V934 Her }\end{array}$ & $\begin{array}{l}2556.931 \\
256.644\end{array}$ & $\begin{array}{l}78.718 \\
23.972\end{array}$ & $\begin{array}{l}\text { Galaxy Cluster } \\
\text { LMXB/NS }\end{array}$ & & $\begin{array}{l}4 \mathrm{U} \quad 1707+78 \\
4 \mathrm{U} \quad 100+24\end{array}$ & \\
\hline 108 & MAXI J1716-629 & 259.2 & -62.9 & 8.8 & 1.6 & 0.2 & NGC 6300 & 259.248 & -62.821 & Sy 2 & 0.0037 & SWIFT J1717.1-6249 & \\
\hline 109 & MAXI J1741+185 & 265.5 & 18.5 & 7.3 & 1.7 & 0.2 & $4 \mathrm{C}+18.51$ & 265.530 & 18.456 & Sy1 & 0.186 & RX J1742.1+1827 & \\
\hline 110 & MAXI J17 & 268.4 & -1.4 & 130.1 & 64.2 & 0.5 & SWIFT J1753.5-0127 & 268.368 & -1.452 & LMXB/ BHC & & & \\
\hline 111 & MAXI J1807+061 & 271.9 & 6.1 & 7.7 & 1.9 & 0.2 & V0426 Oph & 271.966 & 5.862 & CV/Dwarf Nova & & 1RXS J180751.8+055144 & \\
\hline 112 & MAXI J1815+498 & 274.0 & 49.8 & 31.3 & 8.7 & 0.3 & AM Her & 274.055 & $\begin{array}{r}49.868 \\
37.105\end{array}$ & CV/AM Her & & $4 \mathrm{U} 1813+50,4 \mathrm{U} 1$ & \\
\hline $\begin{array}{l}113 \\
114\end{array}$ & $\begin{array}{l}\text { MAXI J1825-371 } \\
\text { MAXI J1826+305 }\end{array}$ & $\begin{array}{l}276.4 \\
276.5\end{array}$ & $\begin{array}{r}-37.1 \\
30.6\end{array}$ & $\begin{array}{r}76.9 \\
7.9\end{array}$ & $\begin{array}{r}32.4 \\
1.8\end{array}$ & $\begin{array}{l}0.4 \\
0.2\end{array}$ & $\begin{array}{l}\text { V0691 CrA } \\
\text { CIZA J1825.3+3026 }\end{array}$ & $\begin{array}{l}276.445 \\
276.353\end{array}$ & $\begin{array}{r}-37.105 \\
30.442\end{array}$ & $\begin{array}{l}\text { LMXB } \\
\text { Galaxy Cluster }\end{array}$ & 0.065 & $4 \mathrm{U} 1822-371,4 \mathrm{U} 1822-37$ & \\
\hline 115 & MAXI J1835+328 & 278.8 & 32.8 & 11.5 & 2.7 & 0.2 & $3 \mathrm{C} 382$ & 278.764 & 32.696 & Sy 1 & 0.05787 & H $1832+325$ & \\
\hline 116 & MAXI J1835-328 & 278.9 & -32.9 & 23.0 & 7.3 & 0.3 & XB $1832-330(\mathrm{NGC} 6652)$ & 278.934 & -32.991 & LMXB/NS & & NG & \\
\hline & MAXI J183 & 279.5 & -65.3 & 12.0 & 2.3 & 0.2 & ESO 103-G35 & 279.585 & -65.428 & Sy2 & 0.01329 & H 18 & \\
\hline 118 & MAXI J1839+798 & 279.9 & 79.9 & 13.6 & 2.8 & 0.2 & 3C 390.3 & 280.538 & 79.771 & Sy1 & 0.0561 & $4 \mathrm{U} 1847+78$ & \\
\hline 119 & MAXI J1851-783 & 282.9 & -78.3 & 8.5 & 1.5 & 0.2 & 2MASX J18470283-7831494 & 281.762 & -78.530 & Sy 1 & 0.07412 & $4 \mathrm{U} 1916-79$ & \\
\hline 120 & $\begin{array}{l}\text { MAXI J1855-311 } \\
\text { MAXI J1900-249 }\end{array}$ & $\begin{array}{l}283.8 \\
285.0\end{array}$ & $\begin{array}{l}-31.2 \\
-24.9\end{array}$ & $\begin{array}{l}21.4 \\
68.8\end{array}$ & 6.5 & 0.3 & $\begin{array}{l}\text { V1223 Sgr } \\
\text { Sgr }\end{array}$ & $\begin{array}{r}283.759 \\
285036\end{array}$ & -31.163 & CV/DQ Her & & $4 \mathrm{U} 1849-31$ & \\
\hline $\begin{array}{l}121 \\
122\end{array}$ & $\begin{array}{l}\text { MAXI J1900-249 } \\
\text { MAXI J1920-586 }\end{array}$ & $\begin{array}{l}285.0 \\
290.1\end{array}$ & $\begin{array}{l}-24.9 \\
-58.6\end{array}$ & $\begin{array}{l}68.8 \\
11.8\end{array}$ & $\begin{array}{r}27.2 \\
2.5\end{array}$ & $\begin{array}{l}0.4 \\
0.2\end{array}$ & $\begin{array}{l}\text { HETE J1900.1-2455 } \\
\text { ESO } 141-G 55\end{array}$ & $\begin{array}{l}285.036 \\
290.309\end{array}$ & $\begin{array}{l}-24.921 \\
-58.670\end{array}$ & $\underset{\mathrm{Sy} 1}{\mathrm{LMSP}}$ & 0.036 & $4 \mathrm{U} 1$ & \\
\hline 123 & MAXI J1921+439 & 290.3 & 4 & & 9.7 & 0.3 & Abell 2319 & 290.189 & 43.962 & Galaxy Cluster & 0.0557 & $4 \mathrm{U} 1$ & \\
\hline 124 & MAXI J1924+501 & 29 & 5 & & 2.4 & 0.2 & $\mathrm{CH}$ Cyg & 291 & 50.241 & Symb/WD & & $192433.0+501415$ & \\
\hline 125 & MAXI J1941-104 & 295.4 & -10.4 & 14.1, & 3.8 & 0.3 & $\begin{array}{l}\text { NGC } 6814 \\
\text { V1432 Aql }\end{array}$ & $\begin{array}{l}295.669 \\
295.048\end{array}$ & $\begin{array}{l}-10.323 \\
-10.424\end{array}$ & $\begin{array}{l}\text { Sy1 } \\
\text { CV/AM Her }\end{array}$ & 0.005214 & $\begin{array}{l}\text { 2E } 1939.9-1026 \\
\text { RX J1940.1-1025 }\end{array}$ & \\
\hline 126 & MAXI J1959+650 & & 65.1 & 20.3 & 4.9 & 0.2 & 1ES $1959+650$ & $\begin{array}{l}29.040 \\
29999\end{array}$ & $\begin{array}{r}-10.424 \\
65.148\end{array}$ & BL Lac & 0.04 & QSO B $1959+650$ & \\
\hline 127 & MAXI J2009- & & & 8.0 & 1.6 & 0.2 & NGC 6860 & 302.195 & -61.100 & Sy1 & 0.01488 & RX J200847-61055 & \\
\hline 128 & MAXI J2011-568 & 30 & -56.9 & 22.0 & 5.2 & 0.2 & Abell 3667 & 303 & -56 & Galaxy Cluster & 0.0556 & 1ES $2008-57.0$ & \\
\hline 129 & MAXI J2041+750 & 310.4 & 75.0 & 9.3 & 1.8 & 0.2 & $4 \mathrm{C}+74.26$ & 310.655 & 75.134 & Sy1 & 0.104 & XSS J20404+7521 & \\
\hline 130 & MAXI J2044-107 & 311.0 & -10.7 & 12.3 & 3.3 & 0.3 & Mrk 509 & 311.041 & -10.723 & Sy1.2 & 0.0344 & 3A $2041-107$ & \\
\hline
\end{tabular}


Table 1 (cont'd)

\begin{tabular}{|c|c|c|c|c|c|c|c|c|c|c|c|c|c|}
\hline $\begin{array}{l}\text { (1) } \\
\text { No. }\end{array}$ & $\begin{array}{c}(2) \\
\text { MAXI Name }\end{array}$ & $\begin{array}{c}(3) \\
\text { MAXI R.A. }\end{array}$ & $\begin{array}{c}(4) \\
\text { MAXI Decl. }\end{array}$ & $\begin{array}{c}(5) \\
\text { Significance }\end{array}$ & $\begin{array}{l}\text { (6) } \\
\text { Flux }^{\mathrm{a}}\end{array}$ & $\begin{array}{l}(7) \\
\text { Error }^{\mathrm{a}}\end{array}$ & $\begin{array}{c}(8) \\
\text { Counterpart Name }\end{array}$ & $\begin{array}{c}\text { (9) } \\
\text { Ctpt. R.A. }\end{array}$ & $\begin{array}{c}(10) \\
\text { Ctpt. Decl. }\end{array}$ & $\begin{array}{l}(11) \\
\text { Type }^{b}\end{array}$ & $\begin{array}{c}(12) \\
\text { Redshift }^{c}\end{array}$ & $\begin{array}{c}(13) \\
\text { Other Name }\end{array}$ & $\begin{array}{l}\stackrel{(14)}{\text { Note }^{d}} \\
\end{array}$ \\
\hline 131 & MAXI J2129+121 & 322.5 & 12.2 & 42.7 & 12.2 & 0.3 & $4 \mathrm{U} 2129+12$ & 322.493 & 12.167 & LMXB in globular clus & & $4 \mathrm{U} 2127+119$ & \\
\hline 132 & MAXI J2135-626 & 323.8 & -62.7 & 7.4 & 1.5 & 0.2 & $1 \mathrm{RXS} \mathrm{J21}$ & 324.096 & -62400 & Sy1 & 0.0588 & $6 \mathrm{dFGS}$ & \\
\hline 133 & MAXI J2144+383 & $\begin{array}{l}326.2 \\
326.2\end{array}$ & 38.3 & 550.6 & 702.3 & 1.2 & Cyg X-2 & 326.172 & $\begin{array}{r}-02.400 \\
38.322\end{array}$ & LMXB/NS & & $4 \mathrm{U} 2142+38$ & \\
\hline & MAXI J2201-600 & 330.3 & -60.1 & 8.3 & 1.7 & 0.2 & Abell 3827 & 330.483 & -59.949 & Galaxy Cluster & 0.0984 & 1RXS J220157.8-595648 & \\
\hline & MAXI J2202-319 & & -31.9 & 7.6 & 2.0 & 0.3 & NGC 7172 & 330.508 & -31.870 & Sy2 & 0.00868 & SWIFT J2201.8-3152 & \\
\hline 136 & MAXI J2214+125 & $3:$ & 12.6 & 7.2 & 1.7 & 0.2 & RU Peg & 333.511 & 12.703 & CV/Dwarf Nova & & $2 \mathrm{E} 2211.5+1227$ & \\
\hline 137 & MAXI J2218-084 & 334.6 & -8.4 & 12.5 & 3.3 & 0.3 & FO Aqr & 334.481 & -8.351 & CV/DQ Her & & $2 \mathrm{E} 2215.3-0835$ & \\
\hline 138 & MAXI J223: & .0 & -25.9 & 9.3 & 2.5 & 0.2 & NGC 7314 & 338.943 & -26.050 & Sy1.9 & 0.00476 & उA $2233-259$ & \\
\hline 139 & MAXI J2253+163 & 343.5 & 16.3 & 17.0 & 4.4 & 0.3 & $3 \mathrm{C} 454.3$ & 343.491 & 16.148 & Blazar & 0.859 & $1 \mathrm{E} 2251.5+1553$ & \\
\hline 140 & MAXI J2253-177 & 343.5 & -17.7 & 11.8 & 3.0 & 0.2 & MR 2251-178 & 343.524 & -17.582 & Sy 1 & 0.06398 & 2A $2251-179$ & \\
\hline 141 & MAXI J2255-031 & 343.8 & -3.1 & 12.7 & 3.4 & 0.3 & AO Psc & 343.825 & -3.178 & $\mathrm{CV} / \mathrm{DQ}$ Her & 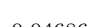 & 3A $2253-033$ & \\
\hline $\begin{array}{l}142 \\
143\end{array}$ & $\begin{array}{l}\text { MAXI } 2305-085 \\
\text { MAXI J2316-425 }\end{array}$ & $\begin{array}{l}346.3 \\
349.1\end{array}$ & $\begin{array}{r}-8.6 \\
-42.6\end{array}$ & $\begin{array}{r}14.8 \\
7.1\end{array}$ & $\begin{array}{l}3.9 \\
2.0\end{array}$ & $\begin{array}{l}0.3 \\
0.3\end{array}$ & $\begin{array}{l}\text { Mrk } 926 \\
\text { Abell S1101 }\end{array}$ & $\begin{array}{l}346.181 \\
348.494\end{array}$ & $\begin{array}{r}-8.686 \\
-42.727\end{array}$ & $\begin{array}{l}\text { Sy1.5 } \\
\text { Galaxy Cluster }\end{array}$ & 0.04686 & 4U $2305-07$ & \\
\hline
\end{tabular}

a The flux is in units of $10^{-11} \mathrm{ergs} \mathrm{cm}^{-2} \mathrm{~s}^{-1}$ in the $4-10 \mathrm{keV}$ band, converted from Crab units; $1 \mathrm{Crab}=1.21 \times 10^{-8} \mathrm{ergs} \mathrm{cm}^{-2} \mathrm{~s}^{-1}$.

bSy: Seyfert galaxy, LMXB: low-mass X-ray binary, HMXB: high-mass X-ray binary, Symb: symbiotic star, NS: neutron star, BHC: black hole candidate, CV: cataclysmic variable, ULX: ultra-luminous

D online database SIMBAD and NED. A blank indicates the source is Galactic, and — indicates the source has no redshift information.

$\mathrm{d}$ (A) Identified by individual inspection since in the image fitting the position is unable to be determined due to significant source confusion with Perseus Cluster. (B) The data is extended compared
with the PSF model. (C) Matsumura et al. (2011) (D)Suzuki et al.(2010) (E) Isobe et al.(2010) (F) This source is likely to be contaminated by $1 \mathrm{RXS}$ J133344.4-231734 and TV Hya. ESO 509-IG66 has double nuclei with similar fluxes in the 2-10 keV band (Guainazzi et al. 2005). The redshift is adopted from Sekiguchi \& Wolstencroft (1992). (G) This source is possibly contaminated by V893 Sco and other nearby sources. 